\title{
Caracterização da primeira ocorrência de anortosito com titanomagnetita vanadífera no Escudo das Guianas, Roraima, Brasil
}

Luis E. A. Goulart' (luis.goulart@cprm.gov.br)

Paulo R. S. Lopes¹ (paulo.lopes@cprm.gov.br)

Marcelo L. Vasquez ${ }^{1}$ (marcelo.vasquez@cprm.gov.br)

Antonio C. S. Oliveira ${ }^{1}$ (charles.oliveira@cprm.gov.br)

${ }^{1}$ Serviço Geológico do Brasil - CPRM, SUREG - MA - Superintendência Regional de Manaus

\begin{abstract}
The present report shows the first occurrence of vanadiferous titanomagnetite (VTM) described in the northern portion of the Amazonian Craton, in Roraima State, Brazil. This occurrence refers to magnetometric and radiometric target explored by SGM - Sociedade Geral de Mineração. Anomalous vanadium concentrations in soil samples complete the characterization of this target. Previous information provided by SGM showed $V_{2} \mathrm{O}_{5}$ concentrations of $0.9 \%$ in the undisclosed drill core interval. The mineralization is associated with dissemination of titanomagnetite in a possible Orosirian anorthosite (VTM - bearing anorthosite). The titanomagnetite showed several lamellar exsolutions of ilmenites. Whole-rock chemical analyses in a core fragment yielded $0.2 \% \mathrm{~V}_{2} \mathrm{O}_{5}$ and $4.1 \% \mathrm{TiO}_{2}$. The results also reveal geochemical fingerprint with flat to slight Eu/Eu* patterns and expressive positive anomaly of Ti. Here, this occurrence highlights the potential for Fe-Ti-V deposits in this Brazilian portion of the Guiana Shield.
\end{abstract}

Keywords: Anorthosite. Guiana Shield. vanadiferous titanomagnetite.

Palavras-chave: Anortosito. Craton Amazonas. titanomagnetita vanadífera.

\section{INTRODUÇÃO}

Os depósitos de vanádio de filiação ortomagmática respondem pelas principais fontes mundiais desse metal (KELLEY et al., 2017). As mineralizações consistem em camadas, lentes e veios com titanomagnetita vanadífera (TMV) disseminada a maciça, associadas a intrusões máfico-ultramáficas acamadadas, intrusões gabro-anortosíticas e maciços anortosíticos, com idades entre o Arqueano e o Mesoproterozoico (EALES; CAWTHORN, 1986; WILSON et al., 1996; CHARLIER et al., 2008; PANG; SHELLNUTT; ZHOU, 2015).

Este trabalho caracteriza uma ocorrência de anortosito com titanomagnetita-vanadífera (TMV), com suporte da petrografia, litogeoquímica e química mineral. O corpo com camada de anortosito hospedeiro está situado no leste do estado de Roraima
(Figura 1A), próximo à divisa com a Guiana, no município de Cantá. A descoberta desse corpo é devida a Sociedade Geral de Mineração (SGM) que gentilmente concedeu ao Serviço Geológico do Brasil - CPRM uma amostra de testemunho de sondagem obtido em trabalhos exploratórios no citado alvo.

\section{CONTEXTO GEOLÓGICO}

A área está localizada no leste do estado de Roraima e faz parte do lado brasileiro do Escudo das Guianas, no norte do Cráton Amazonas (Figura 1A). Esse segmento crustal apresenta um substrato siálico de idade proterozica (FRAGA; ARAÚJO; HADDAD, 1999), representado por metagranitoides e gnaisses da Suite Metamórfica Rio Urubu assim como paragnaisses kinzingíticos do Grupo Cauarane (Figura 1B). Intrudidos nesse substrato siálico, ocorrem corpos 
alongados de mangerito associados à Suíte Serra da Prata (FRAGA; ARAÚJO, 1999) assim como pequenos corpos máfico-ultramáficos correlacionados à Suite Máfico-Ultramáfica Uraricaá (PINHEIRO et al., 1981).

A Suite Serra da Prata é formada predominantemente de charnockito e mangerito, com granito com textura rapakivi subordinado (FRAGA; ARAUJO, 1999). Idades $\mathrm{Pb}-\mathrm{Pb}$ (evaporação em zircão) forneceram idades situadas no intervalo entre $1943 \pm 5 \mathrm{Ma}$ e $1933 \pm 2 \mathrm{Ma}$ (FRAGA et al., 2009). Velasquez (2015) obteve em granito com textura rapakivi milonitizado, interpretado como um segmento deformado dessa unidade, uma idade de cristalização U-Pb SHRIMP em zircão de 1916,7 $\pm 4,5 \mathrm{Ma}$, com uma possível idade de metamorfismo em $1863 \pm 6,7$. Consideramos a datação de 1916 Ma como a melhor idade de cristalização para esta unidade a qual corrobora a existência de um magmatismo orosiriano precursor.

A Suite Máfico-Ultramáfica Uraricaá compreende corpos formados de gabro, gabronorito e hornblendito. Riker et al. (1999) correlacionaram essas rochas aos litotipos congêneres descritos por Pinheiro et al. (1981) em sua área-tipo, na Serra Uraricaá, porção NW do estado de Roraima, onde Fraga et al. (2016) apresentaram uma idade U-Pb SHRIMP em zircão de $1882 \pm 4 \mathrm{Ma}$, obtida em olivina gabro.

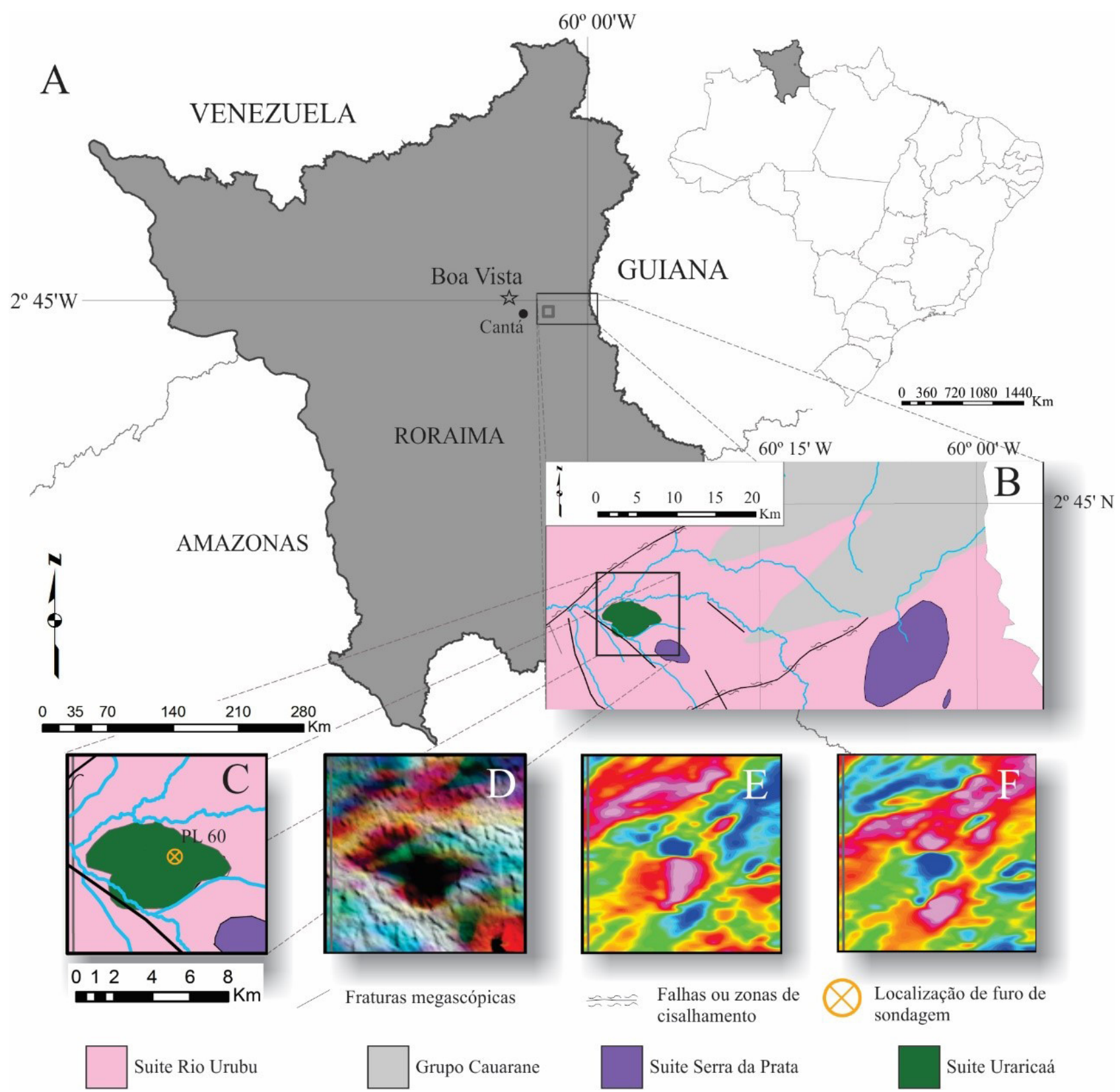

Figura 1 - A) Mapa de localização da área descrita no presente trabalho B) Mapa geológico de um segmento crustal abordando as principais unidades litológicas nas imediações da ocorrência mineral descrita neste trabalho. C) Detalhe da cartografia geológica da ocorrência. Detalhe de mapas aerogeofísicos: D) mapa gamaespectrométrico K-eTh-eU (ternário - RGB); E) mapa magnetométrico de campo magnético anômalo com redução ao polo; F) mapa magnetométrico de campo magnético anômalo. 


\section{DESCRIÇÃO DA OCORRÊNCIA}

\section{Informações relevantes (geologia, geofísica e geoquímica de baixa densidade)}

A ocorrência de anortosito com $\operatorname{TMV}\left(60^{\circ} 23^{\prime}\right.$ $23^{\prime \prime} \mathrm{W} / 2^{\circ} 37^{\prime} 12,6^{\prime \prime} \mathrm{N}$ ) está localizada no município de Cantá, a $23 \mathrm{~km}$ da sede municipal. 0 alvo ocupa uma suave elevação com cerca de 4-5 km, alongada aproximadamente na direção NE-SW. Levantamento geológico regional preliminar cartografou um corpo de mangerito nas proximidades dessa elevação (FRAGA; ARAUJO, 1999).

Aerolevantamentos geofísicos recentes (Figuras $1 \mathrm{C}, 1 \mathrm{D}$ e $1 \mathrm{E}$ ) mostraram que essa colina é coincidente com uma anomalia magnética positiva expressiva, observada em mapa magnetométrico de campo magnético anômalo (CMA) com filtro de redução ao polo (CMA-RP). O levantamento gamaespectrométrico, por sua vez, revelou uma anomalia na composição ternária (RGB) de aspecto grosseiramente zonado, formada na parte norte por um domínio periférico com elevada contagem no canal de $\mathrm{K}$ que decresce em direção ao centro, dando lugar a um domínio gamaespetrométrico intermediário marcado por elevada contagem nos canais de eTh e eU. Esse domínio é identificado pela predominância nas cores entre verde e azul, bem como por um setor central, com baixa contagem em todos os canais, registrado pelas cores escuras tendendo a preto.

No centro da anomalia magnética, coincidindo com o domínio gamaespectrométrico de baixa contagem nos canais de $\mathrm{K}$, eTh e eU, existem dados analíticos de solo provenientes de levantamento geoquímico regional de baixa densidade, que revelaram uma anomalia geoquímica de $\mathrm{V}$ com concentrações de 221 ppm, associadas a concentrações de $P=236$ ppm, $\mathrm{Fe}=12,18 \%$ e $\mathrm{Ti}=0,11 \%$. Uma sondagem rotativa com coroa diamantada realizada pela SGM, sobre esta anomalia geoquímica, interceptou um nível de anortosito com titanomagnetita vanadífera disseminada, a profundidades superiores a 40 metros. Informações verbais da empresa reportaram que análises litogeoquímicas nos testemunhos de sondagem identificaram concentrações de $\mathrm{V}_{2} \mathrm{O}_{5} \approx 0,9 \%$.

\section{Petrografia}

O anortosito com TMV é isotrópico, maciço, denso, fortemente magnético e apresenta cor predominantemente cinza escuro (Figura $2 \mathrm{~A}$ ). A rocha tem granulometria grossa e possui cristais de plagioclásio com 2-3 cm de comprimento (Figura 2B).

A rocha é formada por plagioclásio (65\%), clinopiroxênio (7\%), ortopiroxênio (5\%), magnetita $(15 \%)$, ilmenita (5\%) e calcopirita/pirita/pirrotita (3\%), com biotita e hornblenda não quantificadas (< 1\%). Apatita ocorre como traços e espinélio é raro.

O plagioclásio é prismático, subédrico a anédrico, com predominância estimada de composição labradorita $\left(\mathrm{An}_{50-55}\right)$, indicando tratar-se de um labradorita-anortosito (ANDERSON; MORIN, 1969).

Os cristais de orto e clinopiroxênio apresentam 0,5-1,0 cm de tamanho, são subédricos a anédricos (Figura 2B). O clinopiroxênio é pigeonítico e exibe exsoluções de hiperstênio e ilmenita. Juntamente com plagioclásio, os cristais de orto/clinopiroxênio e magnetita caracterizam textura adcumulática triminerálica a plagioclásio, magnetita e clinopiroxênio.

A magnetita (Figura 2D) ocorre como cristais com 1-2 cm de tamanho, hábito anédrico e exibe exsolução de paletas de ilmenita. Eventualmente, a ilmenita pode ocorrer como grãos isolados maiores, quase sempre em contato com magnetita e/ou agregados de cristais de sulfetos (pirita/pirrotita e calcopirita; Figura 2B). Essa relação sugere que a cristalização dos óxidos ocorreu na presença de uma fase magmática sulfetada remanescente, subsaturada em enxofre.

Cristais zonados de espinélio, em tons de castanho escuro no centro a castanho esverdeado na borda, que sugerem variação composicional de $\mathrm{Cr}$ -pleonasto a pleonasto, foram observados como inclusões nos cristais de magnetita (Figura 2C). Franjas quelifíticas de biotita e hornblenda no contato entre plagioclásio e magnetita ou menos comumente no contato entre plagioclásio e ortopiroxênio indicam reação durante cristalização desses minerais (Figura 2C). Apatita é mais rara e ocorre como inclusões de cristais alongados hospedadas nos cristais de plagioclásio e piroxênios.

\section{Litogeoquímica}

$\mathrm{O}$ anortosito com TMV apresentou caráter ultrabásico com concentração de $\mathrm{SiO}_{2}=41,4 \%$ (Tabela 1), $\mathrm{Al}_{2} \mathrm{O}_{3}=11,1 \%, \mathrm{MgO}=6,52 \%, \mathrm{CaO} \approx 10,0 \%$ e baixas concentrações de álcalis $\left(\mathrm{K}_{2} \mathrm{O}=0,25 \%\right.$ e $\mathrm{Na}_{2} \mathrm{O}=$ $1,93 \%)$. A concentração dos elementos formadores de TMV são igualmente elevadas com FeOt (24,6\%), $\mathrm{TiO}_{2}(4,08 \%)$ e $\mathrm{V}_{2} \mathrm{O}_{5}(0,2 \%)$.

A composição normativa (Tabela 1 ) equivale à de um gabronorito sugerindo a influência da granulação na representatividade da amostragem litogeoquímica. O comportamento dos elementos terras raras no diagrama de ETR (Figura 3B), revelou um padrão ligeiramente horizontalizado (Figura 3), definido por razões $\operatorname{Lan} / \mathrm{Ybn} \approx 1,77, \operatorname{Lan} / \mathrm{Gdn} \approx 1,11$ e $\mathrm{Gdn} / \mathrm{Ybn} \approx$ 1,60. Anomalia Eu/Eu* ligeiramente positiva obtida $(\approx 1,1)$, demonstra que a amostra obtida provém de um nível anortosítico cumulático mais fracionado.

A assinatura geoquímica é também marcada por anomalia positiva de Ti bastante expressiva, caracterizando o caráter cumulático dos óxidos de Fe e $\mathrm{Ti}$. Anomalias positivas de $\mathrm{K}, \mathrm{Ba}, \mathrm{Pb}$ e $\mathrm{Sr}$, bem como anomalia negativa de $\mathrm{P}$, também são observadas ( $\mathrm{Fi}$ gura 3B).

Particularmente, a anomalia de $\mathrm{K}$ reflete a biotita e a hornblenda como fases minerais secundárias, enquanto a anomalia negativa de $\mathrm{P}$ sugere que concentrações mais expressivas de apatita podem ter sido cristalizadas já no início do fracionamento. 


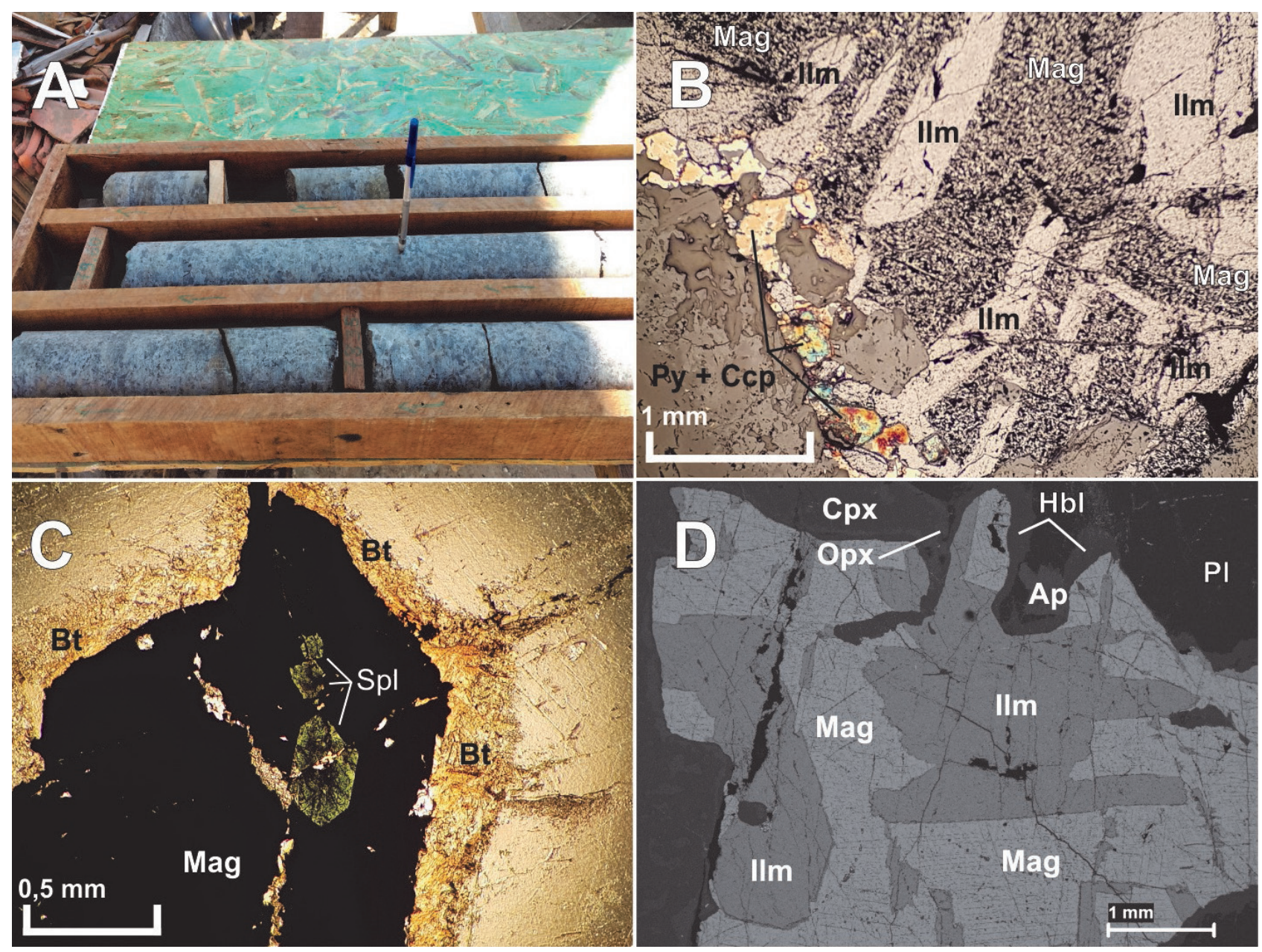

Figura 2 - A) Intervalo dos testemunhos da SGM onde foi coletada a amostra estudada neste trabalho.

B) Fotomicrografia onde é possível observar cristal de magnetita (rosado mais escuro) com exsolução de paletas e cristais maiores de ilmenita (rosado mais claro). Aglomerados de calcopirita, pirita e pirrotita ocorrem comumente em contato ou inclusos com cristais de magnetita (luz refletida, polarizadores //, objetiva 4x). C) Seção com evidência de magnetita com inclusão de cristal de Cr-espinélio. As bordas da magnetita no contato com plagioclásio mostram franja quelifítica formada predominantemente por lamelas de biotita e hornblenda (luz transmitida, polarizadores //, objetiva 10 X. D) Imagem BSE de cristal de magnetita com exsolução de lamelas de ilmenita. Abreviações: Ap - apatita; Bt - biotita; Ccp calcopirita; Cpx - clinopiroxênio; Ilm - ilmenita; Mag - magnetita; Opx - ortopiroxênio; Pl - plagioclásio; Py - pirita.

Tabela 1 - Composição química e normativa do anortosito com TMV (amostra PL 60).

\begin{tabular}{|c|c|c|c|c|c|c|c|c|c|c|c|c|c|c|c|c|c|c|c|}
\hline \multicolumn{12}{|c|}{ Óxidos (\%) } & & & & & & & & \\
\hline $\mathrm{SiO}_{2}$ & $\mathrm{TiO}_{2}$ & $\mathrm{Al}_{2} \mathrm{O}_{3}$ & $\mathrm{Fe}_{2} \mathrm{O}_{3}$ & $\mathrm{MnO}$ & $\mathrm{MgO}$ & $\mathrm{CaO}$ & $\mathrm{Na}_{2} \mathrm{O}$ & $\mathrm{K}_{2} \mathrm{O}$ & $\mathrm{P}_{2} \mathrm{O} 5$ & LOI & Total & & & & & & & & \\
\hline 41,65 & 4,1 & 11,17 & 24,75 & 0,26 & 6,56 & 10,06 & 1,94 & 0,25 & 0,05 & $-0,54$ & 100,25 & & & & & & & & \\
\hline \multicolumn{20}{|c|}{ Elementos maiores, menores e traços (ppm) } \\
\hline $\mathrm{Zr}$ & $\mathrm{Hf}$ & Y & U & Th & $\mathrm{Nb}$ & $\mathrm{Ta}$ & $\mathrm{Ba}$ & Ga & $\mathrm{Rb}$ & $\mathrm{Sr}$ & Co & Sc & $\mathrm{v}$ & $\mathrm{Cu}$ & Mo & $\mathrm{Ni}$ & $\mathrm{Pb}$ & $\mathrm{Zn}$ & $\mathrm{Cr}$ \\
\hline 56,5 & 1,66 & 18,5 & 0,08 & 0,4 & 2,7 & 0,34 & 96 & 34,9 & 2,1 & 264,9 & 97,9 & 75,4 & 964 & 214 & 0,11 & 65 & 1,6 & 1,78 & $<1$ \\
\hline \multicolumn{14}{|c|}{ Elementos de terras raras (ppm) } & \multicolumn{4}{|c|}{ Razões normalizadas de ETR } & & \\
\hline La & $\mathrm{Ce}$ & $\mathrm{Pr}$ & $\mathrm{Nd}$ & $\mathrm{Sm}$ & $\mathrm{Eu}$ & $\mathrm{Gd}$ & $\mathrm{Tb}$ & Dy & Ho & $\mathrm{Er}$ & $\mathrm{Tm}$ & $\mathrm{Yb}$ & Lu & $\mathrm{Eu} / \mathrm{Eu}^{*}$ & $(\mathrm{La} / \mathrm{Yb})_{\mathrm{N}}$ & $(\mathrm{Ga} / \mathrm{Yb})_{\mathrm{N}}$ & $(\mathrm{La} / \mathrm{Ga})_{\mathrm{N}}$ & & \\
\hline 4,2 & 10,1 & 1,67 & 8,6 & 2,8 & 1,07 & 3,28 & 0,51 & 3,42 & 0,63 & 1,75 & 0,24 & 1,7 & 0,23 & 1,08 & 1,77 & 1,6 & 1,11 & & \\
\hline \multicolumn{10}{|c|}{ Composição normativa CIPW (\%) } & & & & & & & & & & \\
\hline $\mathrm{PI}$ & Or & $\mathrm{Di}$ & Hy & ॥ & Mt & $A p$ & $\mathrm{Zr}$ & Py & Total & & & & & & & & & & \\
\hline 37,56 & 1,55 & 23,38 & 14,47 & 7,82 & 14,4 & 0,12 & 0,01 & 0,61 & 99,97 & & & & & & & & & & \\
\hline
\end{tabular}



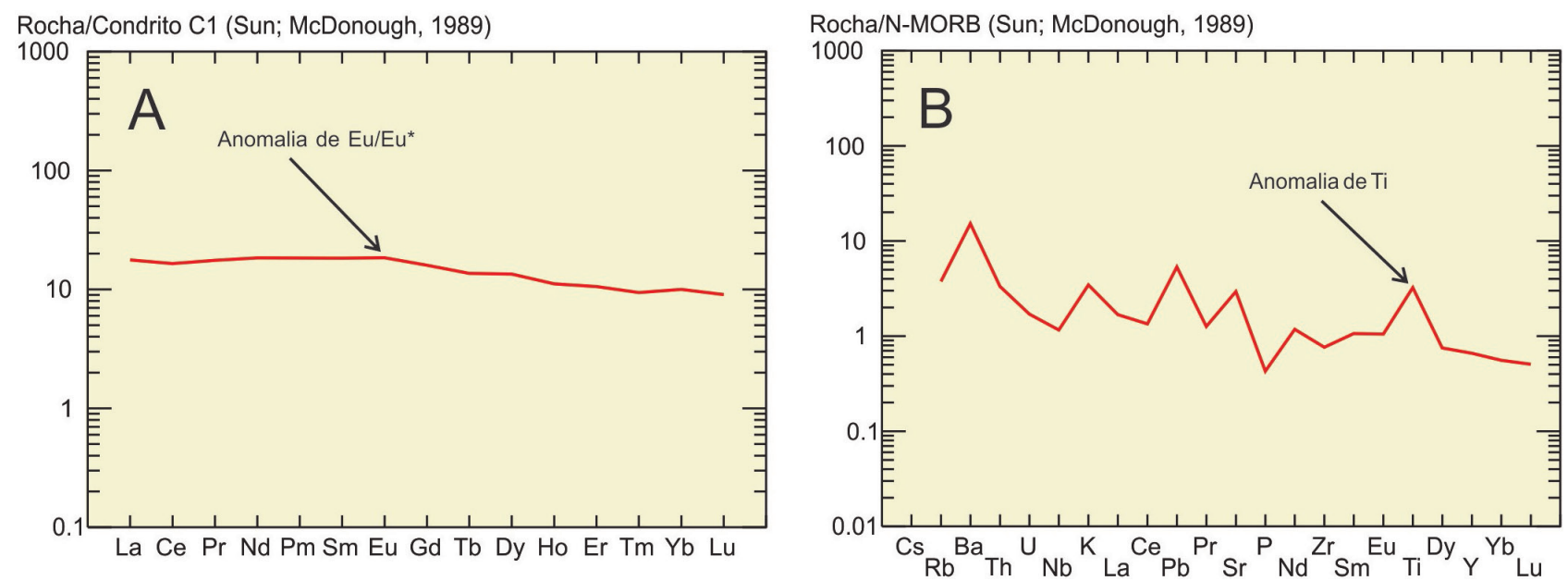

Figura 3 - A) Distribuição de elementos terras raras, normalizada ao condrito C1 (SUN; McDONOUGH, 1989). B) Diagrama multielementar normalizado ao N-MORB (SUN; MCDONOUGH, 1989).

\section{Química mineral}

Dados analíticos de MEV-EDS foram obtidos no LAMIN-BE/CPRM em seis cristais de magnetita (26 pontos analíticos) e oito cristais de ilmenita (24 pontos analíticos) e estão apresentados na Tabela 2. Os cristais de magnetita analisados (Figura 2D) revelaram uma concentração média de $\mathrm{V} \approx 0,8-1,55 \%\left(\mathrm{~V}_{2} \mathrm{O}_{5}\right.$ $\approx 1,42-2,37 \%$ ) e $\mathrm{Fe} \approx 72,01-78,17 \%$, confirmando a mineralização como TMV. Entretanto, as microanálises também apontam a ilmenita como mineral de minério de vanádio, além de titânio. As concentrações médias desses metais contidos na ilmenita foram $\mathrm{V}$ $\approx 0,40-0,99 \%\left(\mathrm{~V}_{2} \mathrm{O}_{5} \approx 0,7-1,76 \%\right)$ e $\mathrm{Ti} \approx 28,25-33,51 \%$.

Tabela 2 - Síntese de resultados analíticos de EDS obtidos em cristais de magnetita e ilmenita da amostra PL-60.

\begin{tabular}{|c|c|c|c|c|}
\hline Mineral & $\begin{array}{c}\mathbf{V} \\
\text { Peso\% }\end{array}$ & $\begin{array}{c}\mathbf{V}_{2} \mathbf{O}_{5} \\
\text { (equivalente)\% }\end{array}$ & $\begin{array}{c}\mathbf{F e} \\
\text { Peso\% }\end{array}$ & $\begin{array}{c}\mathbf{T i} \\
\text { Peso\% }\end{array}$ \\
\hline Magnetita & $0,80-1,55$ & $1,42-2,37$ & $72,01-78,17$ & - \\
\hline Ilmenita & $0,40-0,99$ & $0,7-1,76$ & $30,98-37,68$ & $28,25-33,51$ \\
\hline
\end{tabular}

\section{DISCUSSÕES E CONCLUSÕES}

A assinatura gamaespectrométrica e magnetométrica obtida sobre a ocorrência de TMV mostrada no presente trabalho é consistente com a assinatura de corpos charnockíticos e gabro-anortosíticos existentes na região.

A assinatura geofísica associada ao outlier geoquímico obtido em análise de solo, por sua vez, mostrou ser um critério razoável para sugerir alvos potenciais para mineralizações de Fe-Ti-V. A rocha com TMV interceptada em furo de sondagem, caracterizada como labradorita-anortosito, define um importante metalotecto para prospeç̧ão regional: os anortositos a labradorita em geral hospedam titanomagnetita e ilmenita enquanto os anortositos a andesina hospedam hematita e hemoilmenita (ANDERSON; MORIN, 1969).
Os resultados litogeoquímicos e petrográficos obtidos indicam o plagioclásio e os óxidos de Fe e $\mathrm{Ti}$ (magnetita vanadífera e ilmenita) como principais minerais da assembleia fracionante, onde a cristalização fracionada e a química do magma primordial seriam os controles preponderantes da mineralização nesta ocorrência. O fracionamento do plagioclásio é demonstrado pelas anomalias positivas de $\mathrm{Ba}, \mathrm{Pb}$ e Sr. Nesse sentido, a anomalia levemente positiva de $\mathrm{Eu} / \mathrm{Eu}^{*}$ indica que grande parte do Eu já teria ficado retida nos primeiros cumulatos. A anomalia positiva de $\mathrm{Ti}$, bastante expressiva na rocha, sustenta que os óxidos de Fe e Ti formaram uma fase cumulática de titanomagnetita e ilmenita no anortosito estudado. Em particular, a presença de anomalia positiva de Ti nos padrões geoquímicos pode ser usada como critério para identificação de possíveis unidades mineralizadas. Por outro lado, a presença da anomalia negativa de P observada não exclui que níveis mineralizados em apatita ocorram em outras litofácies do corpo anortosítico.

Apesar dos resultados obtidos suportarem um controle magmático importante para esta mineralização, a correlação entre esta ocorrência e as unidades geológicas conhecidas na região é incerta. Atualmente os dados geológicos disponíveis não permitem definir a unidade portadora de TMV, sugerindo apenas uma correlação com corpos ou camadas de anortosito associados à Suite Serra da Prata ou Suite Máfico-Ultramáfica Uraricaá, que nunca foram efetivamente identificados em mapeamento de superfície. Dessa forma, é possível que essa ocorrência de anortosito com TMV represente uma porção de corpos gabro-anortosíticos sub-aflorantes correlacionados às litologias conhecidas na região, mas que ainda não foram identificados, ou mesmo a um novo metalotecto representado por uma nova unidade, que até o momento não foi descrita. Novos estudos isotópicos, geoquímicos e geocronológicos são recomendados para melhor caracterizar a mineralização e suas rochas hospedeiras. 
Com relação ao potencial desta mineralização, as análises pontuais indicam a existência de uma unidade anortosítica hospedeira de TMV no estado de Roraima com $\approx 14,5 \%$ de magnetita (@ 0,8-1,55\% V) e $\approx 8 \%$ de ilmenita (@ 0,5-0,99\% V; @ 28,25-33,51\% Ti) com projeção calculada para o intervalo do testemunho analisado de $65 \%$ de magnetita e $35 \%$ de ilmenita. Tanto as concentrações de vanádio no minério informadas pela SGM $\left(\mathrm{V}_{2} \mathrm{O}_{5} \approx 0,9 \%\right)$ quanto as concentrações obtidas nesse estudo $\left(\mathrm{V}_{2} \mathrm{O}_{5} \approx 0,2 \%\right)$ correspondem a valores economicamente lavráveis em muitos depósitos de TMV de classe mundial conhecidos (S $\varnothing$ RENSEN et al., 2016; KELLEY et al., 2017). Com valores de $\mathrm{V}_{2} \mathrm{O}_{5}$ na China atingindo em agosto de 2019 (fonte: https://www.vanadiumprice. com/) a casa de US\$ 8,91/Lb (US\$ 19.621,00/ton) e o ferro-vanádio a $80 \%$ sendo cotado a US\$ 38.500,00/ ton, o estado de Roraima desponta como uma importante oportunidade de investimento em pesquisa mineral para esses metais.

\section{REFERÊNCIAS}

ANDERSON, A. T.; MORIN, M. Two types of massif anorthosite and their implications regarding the thermal history of the crust. In: Isachsen, Y. W. (ed.). Origin of anorthosite and related rocks. Albany, New York: New York State Museum and Science Service Memoir, 1969. v. 18, p. 57-69.

CHARLIER, Bernard; SAKOMA, Emmanuel; SUAVÉ, Martin; STAN $\neg$ AWAY, Kerry; AUWERA, Jacqueline Vander; DUCHESNE, Jean-Clair. The Grader layered intrusion (Havre-Saint-Pierre Anorthosite, Quebec) and genesis of nelsonite and other Fe-Ti-P-ores. Lithos, Amsterdam, v. 101, n. 3-4, p. 359-378, 2008.

EALES, H. V.; CAWTHORN, R. G. The Bushveld Complex. In: CAWTHORN, R. G. (ed.). Layered intrusions. Amsterdam: Elsevier, 1996. p. 181-229.

FRAGA, Lêda Maria; ARAÚJO, Roberto Vieira de; HADDAD, Regina Clélia. Suíte Metamórfica Rio Urubu (Pru). In: REIS, Nelson Joaquim; FRAGA, Lêda Maria; ARAÚJO, Roberto Vieira de; RIKER, Roberto Lopes; AILMEIDA, Marcelo Esteves de; HADDAD, Regina Clélia; MILLIOTI, Cláudio; CARVALHO, José Maria de Azevedo. Roraima Central, Folhas NA.20-X-B e NA.20-X-D (inteiras), NA.20-X-A, NA.20-X-C, NA.21-V-A e NA.21-V-C (parciais). Escala 1:500.000. Estado do Amazonas. Brasília: CPRM, 1999. p. 38-49. Programa Levantamentos Geológicos Básicos - PLGB.

FRAGA, Lêda Maria; ARAÚJO, Roberto Vieira de. Suíte Intrusiva Serra da Prata (Msp). In: REIS, Nelson Joaquim; FRAGA, Lêda Maria; ARAÚJO, Roberto Vieira de; RIKER, Roberto Lopes; AILMEIDA, Marcelo Esteves de; HADDAD, Regina Clélia; MILLIOTI, Cláudio; CARVALHO, José Maria de Azevedo. Roraima Central, Folhas NA.20-X-B e NA.20-X-D (inteiras), NA.20-X-A, NA.20-X-C, NA.21-V-A e NA.21-V-C (parciais). Escala 1:500.000. Estado do Amazonas. Brasília: CPRM, 1999. p. 83-89. Programa Levantamentos Geológicos Básicos - PLGB.
FRAGA, Lêda Maria; DREHER, Ana Maria; REIS, Nelson Joaquim; SCANDOLARA, Jaime Estevão. Litoestratigrafia. In: FRAGA, Lêda Maria; DREHER, Ana Maria; Reis, Nelson Joaquim; BETIOLLO, Leandro Menezes; SCANDOLARA, Jaime Estevão. (org.). Geologia e recursos minerais da folha Ilha de Maracá - NA.20-X-A, Estado de Roraima, escala 1:250.000. Manaus: CPRM, 2016. p 35-36. Programa Geologia do Brasil - PGB.

FRAGA, Lêda Maria; MACAMBIRA, Moacir José Buenano; DALL'AGNOL, Roberto; COSTA, João Batista Sena. 1.94-1.93 Ga charnockitic magmatism from the central part of the Guyana Shield, Roraima, Brazil: single-zircon evaporation data and tectonic implications. Journal of South American Earth Sciences, Amsterdam, v. 27, n. 4, p. 247-257, 2009.

KELLEY, Karen D.; SCOTT, Clinton T.; POLYAK, Désirée E.; KIMBALL, Bryn E. Vanadium. In: SCHULZ, Klaus J.; DEYOUNG JUNIOR, John H.; SEAL II, Robert R.; BRADLEY, Dwight C. (ed.). Critical mineral resources of the United States: economic and environmental geology and prospects for future supply. Reston, Virginia: U.S. Geological Survey, 2017. p. 797-832. (U.S. Geological Survey Professional Paper, 1802-U).

PANG, K. N.; SHELLNUTT, J. G.; ZHOU, M. F. The Panzhihua Intrusion, SW China. In: CHARLIER, Bernard; NAMUR, Olivier; LATYPOV, Rais; TEGNER, Christian. (ed.). Layered intrusions. Dordrecht: Springer, 2015. p. 435-464.

PINHEIRO, Sandoval da Silva; COSTI, Hilton Tulio; SANTOS, João Oreste S.; PESSOA, Manoel Roberto; MENEZES, Ricardo Gallart de; RIKER, Silvio R. Lopes. Projeto Catrimâni-Uraricoera. Manaus: CPRM, 1981. v. 1 , p. 100-133.

RIKER, Silvio Roberto Lopes; ARAUJO, Roberto Vieira de; MILLIOTI, Cláudio; REIS, Nelson Joaquim. Suíte Máfico-Ultramáfica Uraricaá (Mu). In: REIS, Nelson Joaquim; FRAGA, Lêda Maria; ARAÚJO, Roberto Vieira de; RIKER, Roberto Lopes; AILMEIDA, Marcelo Esteves de; HADDAD, Regina Clélia; MILLIOTI, Cláudio; CARVALHO, José Maria de Azevedo. Roraima Central, Folhas NA.20-X-B e NA.20-X-D (inteiras), NA.20-X-A, NA.20-X-C, NA.21-V-A e NA.21-V-C (parciais). Escala 1:500.000. Estado do Amazonas. Brasília: CPRM, 1999. p. 72-73. Programa Levantamentos Geológicos Básicos - PLGB.

SØRENSEN, Lars Lund; WEATHERLEY, San; KOKFELT, Thomas; NIELSEN, Troels. Magmatic titanium-vanadium potential in Greenland. Geology and Ore: Exploration and Mining in Greenland, Greenland, n. 27, p. 2-13, 2016.

SUN, S. S.; McDONOUGH, W. F. Chemical and isotopic systematics of oceanic basalts: implications for mantle composition and processes. In: SAUNDERS, A. D.; NORRY, M. J. (ed.). Magmatism in the Ocean Basins. Oxford: The Geological Society, 1989. p. 313-345. (Special Publication, n. 42.). 
VELASQUEZ, C. A. T. Análise das petrotramas das rochas charnockiticas da Serra da Prata, Mucajaí, RR. 2015. 197 f. Dissertação (Mestrado). - Instituto de Ciências Exatas. Universidade Federal do Amazonas, Manaus, 2015.
WILSON, J. R.; ROBINS, B.; NIELSEN, F. M.; DUCHESNE, J. C; VANDER AUWERA, J. The Bjerkreim-Sokndal Layered Intrusion, Southwest Norway. I. In: CAWTHORN, R. G. (ed.) Layered intrusions. Amsterdam: Elsevier Science B.V., p. 231-255, 1996.

\section{INFORME TÉCNICO N 15}

Brasília, setembro de 2019.

ISSN: $2448-2242$

Publicação on-line seriada

Serviço Geológico do Brasil - CPRM

Disponível em: www.cprm.gov.br

\section{Serviço Geológico do Brasil - CPRM}

SBN - Quadra 02 - Bloco H, Ed. Central Brasília, $1^{\circ}$ andar Brasília - DF - Brasil

CEP: 70040-904

Telefone:(61) 2108-8400

www.cprm.gov.br

contatos: seus@cprm.gov.br evandro.klein@cprm.gov.br
Diretor de Geologia e Recursos Minerais José Leonardo Silva Andriotti

\section{Corpo Editorial}

Evandro Luiz Klein (Editor)

João Henrique Larizzatti

Luiz Gustavo Rodrigues Pinto

\section{Revisores}

Luiz Gustavo Rodrigues Pinto

Geysson de Almeida Lages

Normalização Bibliográfica

Nelma Fabrícia da P. Ribeiro Botelho

Editoração Eletrônica

Marcelo Henrique Borges Leão Nelma Fabrícia da P. Ribeiro Botelho
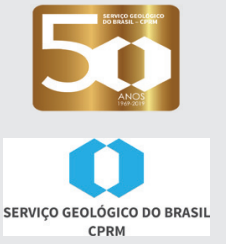